\title{
Rare Case of a Transverse Colon Schwannoma
}

\author{
Mridul Pansari ${ }^{1}$, Daud Lodin ${ }^{1}$, Anupam K. Gupta ${ }^{1}$, Thomas Genuit ${ }^{1}$, Jordan Moseson ${ }^{1}$ \\ 1. Surgery, Charles E. Schmidt College of Medicine, Florida Atlantic University, Boca Raton, USA
}

Corresponding author: Daud Lodin, drdaudlodin@gmail.com

\section{Abstract}

Schwannomas are tumors comprised of schwann cells of the peripheral nervous system and infrequently present in the gastrointestinal tract. Transverse colon schwannomas are an even more rare subtype of gastrointestinal schwannomas. This study presents the case of a transverse colon schwannoma, in addition to presenting a literature review.

Categories: Pathology, Gastroenterology, General Surgery

Keywords: schwannoma, laparoscopic surgery, pathology, colon, hemicolectomy, tumor, transverse colon, surgery, colorectal surgery

\section{Introduction}

Schwannomas are the tumors originating from schwann cells and their presentation as a gastrointestinal (GI) tumor is very rare, making up a small minority of all GI tumors [1]. Along the GI tract, schwannomas are most commonly found in the stomach and present incidentally during endoscopic examination or computer tomography (CT) scans [2-5]. Colorectal schwannomas are even rarer and patients are typically asymptomatic, but can present with signs of obstruction and rectal bleeding [5].

This case report discusses the findings of a transverse colon schwannoma and discusses the epidemiology, histopathology, differential diagnoses, and treatment of this rare tumor.

\section{Case Presentation}

The patient is a 64-year-old female with no significant past medical history, who underwent age appropriate screening colonoscopy and was found to have a submucosal lesion in the transverse colon (Figure 1).

Received 12/27/2019

Review began 04/05/2020

Review ended 04/05/2020

Published 04/09/2020

๑) Copyright 2020

Pansari et al. This is an open access

article distributed under the terms of the Creative Commons Attribution License CC-BY 4.0., which permits unrestricted use, distribution, and reproduction in any medium, provided the original author and source are credited.

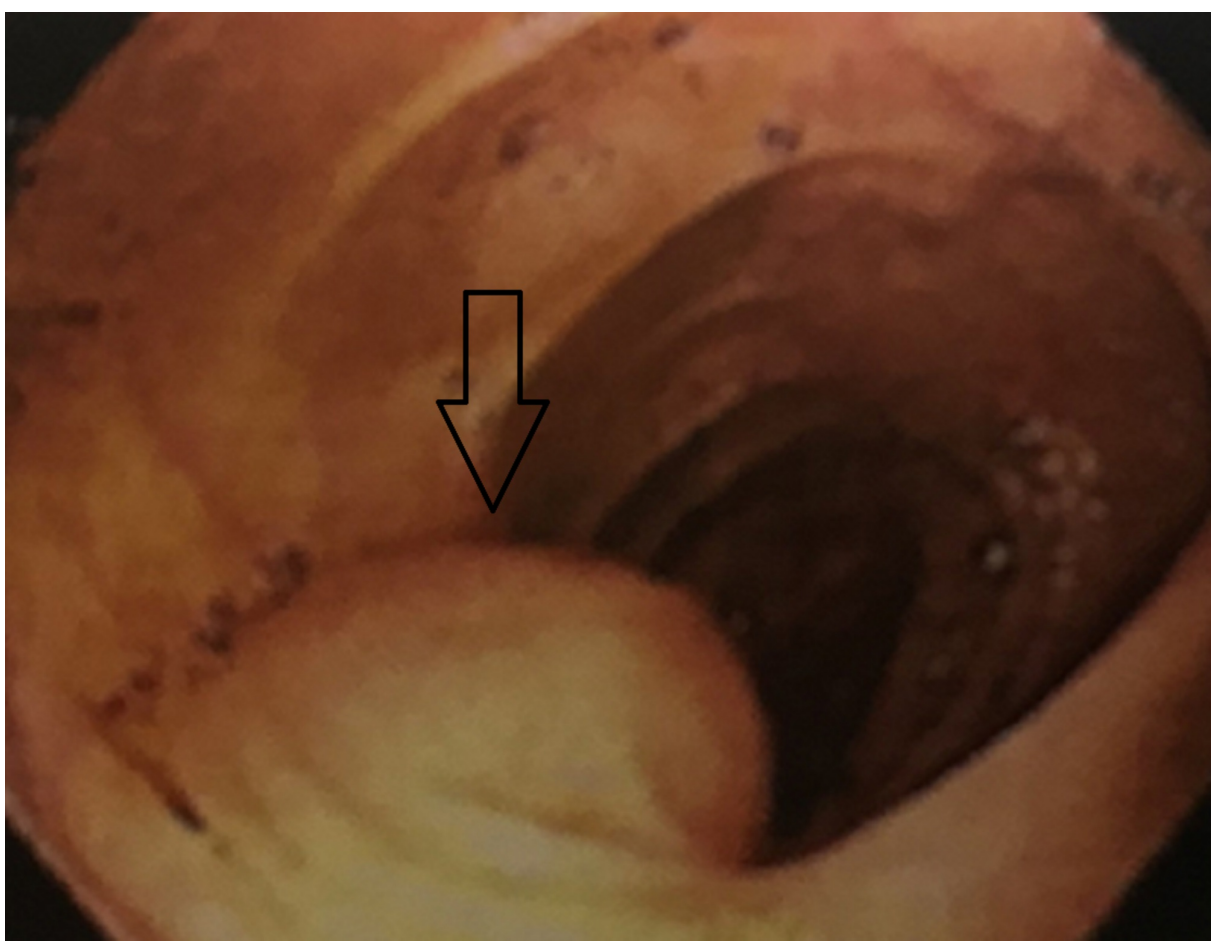

FIGURE 1: Colonoscopic view of a submucosal mass in the transverse colon. 


\section{Cureus}

The mass was tattooed and biopsied. Pathological analysis was unable to diagnose the mass. The patient underwent a computed tomography (CT) scan of her abdomen and pelvis, which demonstrated a 2.1-cm mass in the transverse colon (Figure 2).

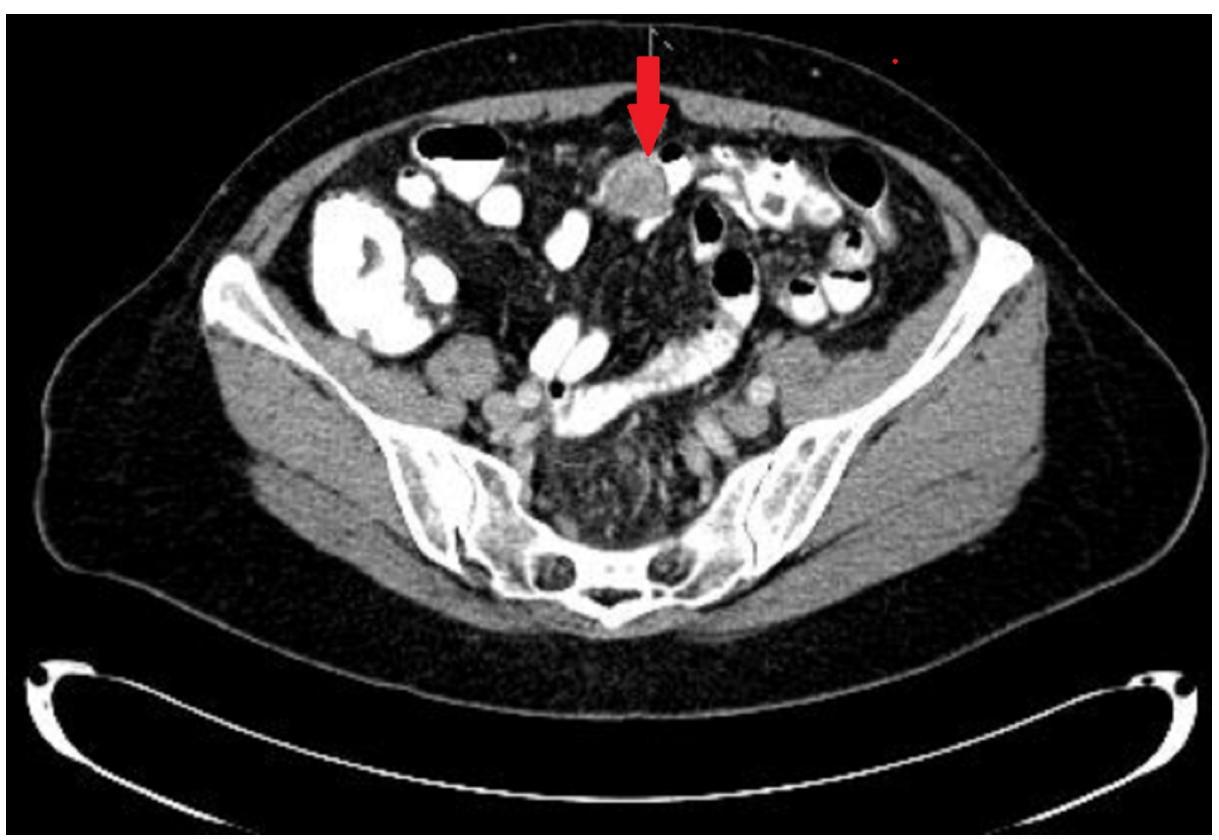

FIGURE 2: Computer tomography scan of the abdomen, indicating a transverse colon mass $2.0 \times 2.1 \mathrm{~cm}$ in size.

The patient was scheduled for a laparoscopic extended right hemicolectomy. The procedure was completed successfully with the resection of the right colon and partial resection of the transverse colon with a side-toside, functional end-to-end terminal ileum to transverse colon anastomosis. The patient had an uneventful postoperative course and was discharged on postoperative day 3 after she tolerated a regular diet. She was seen in clinic two weeks following surgery at which time she denied any complaints. The final pathology report revealed a spindle cell tumor (Figure 3) that stained positive for SOX10 and S-100 (Figures 4, 5) and negative for DOG1, SMA, or CD 117 (Figure ), consistent with a diagnosis of schwannoma. The Ki-67 index was reported to be $1-2 \%$, which would identify this as a benign lesion. The tumor's capsule was intact without evidence of invasion of the tumor (Figure 7). 


\section{Cureus}

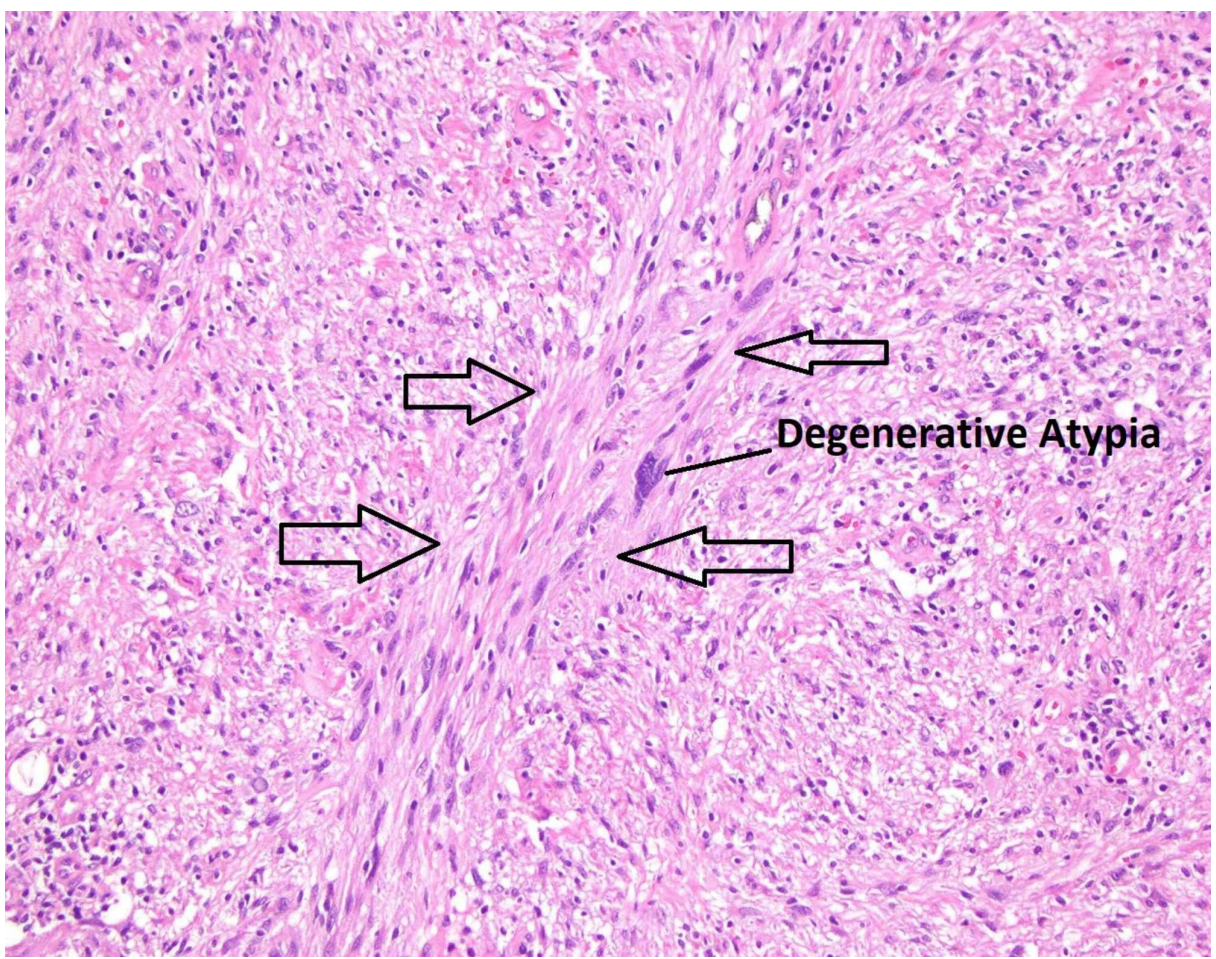

FIGURE 3: Pathology slide of tumor with haematoxylin and eosin staining at 20x magnification. The arrows point to areas of dense spindle cells with interlacing fascicles. The blue area of density at the center of this image is significant for degenerative atypia, which is associated with schwannomas.

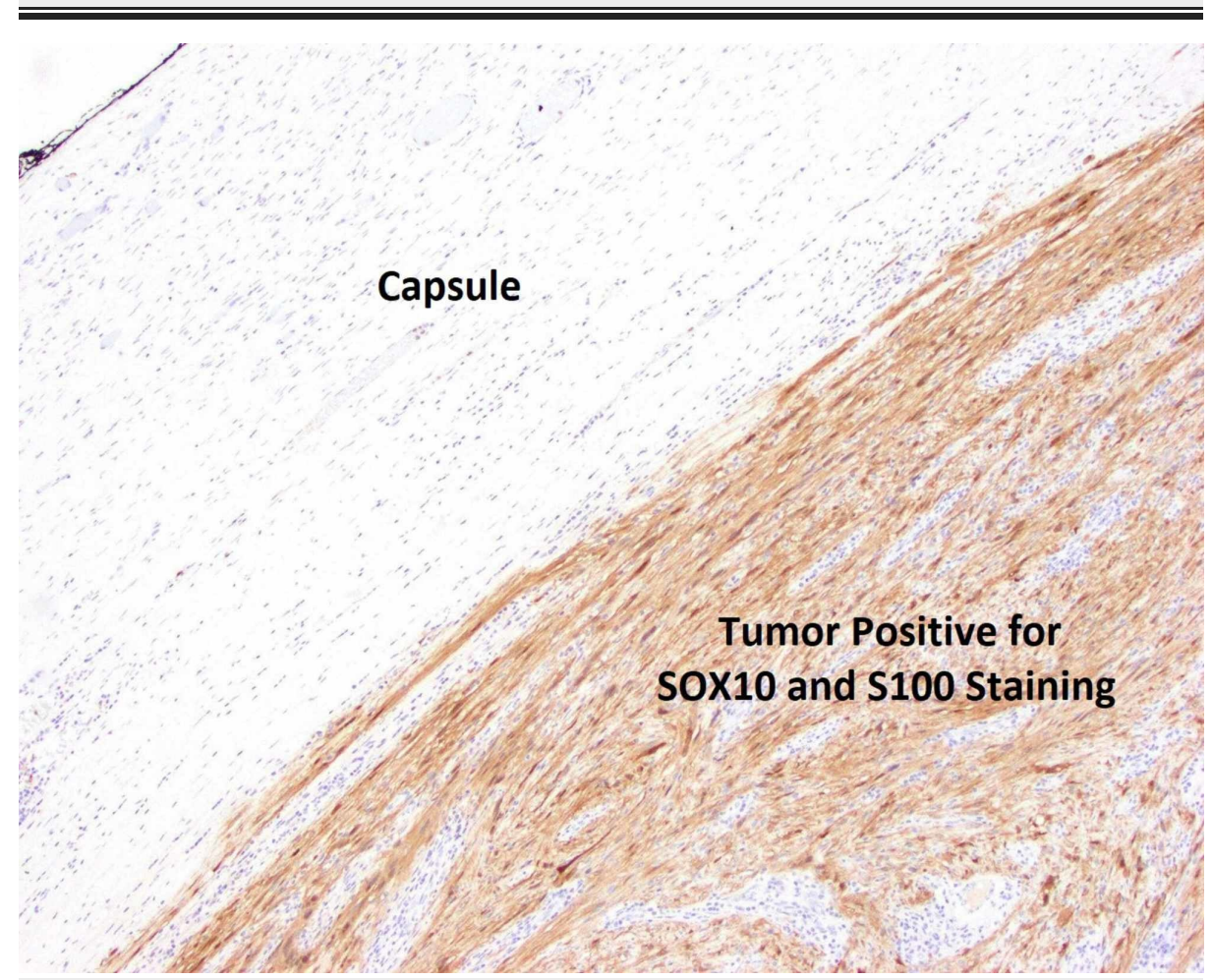

FIGURE 4: Pathology slide of tumor and capsule with SOX10 and S100 positive staining at $4 \mathrm{x}$ magnification. Note that the SOX10 and $\mathrm{S} 100$ staining appear dark brown in consistency. 


\section{Cureus}

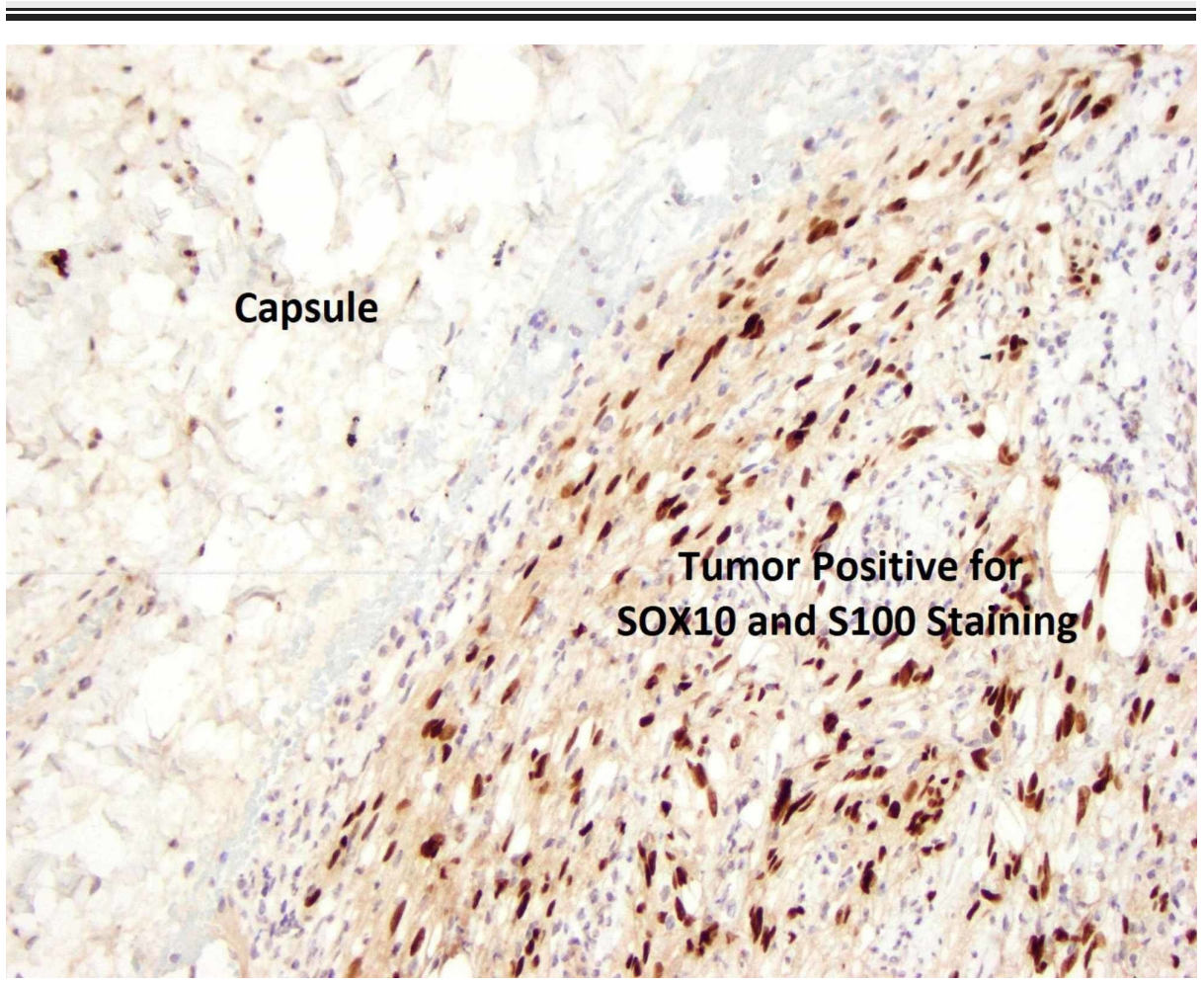

FIGURE 5: Pathology slide of tumor with SOX10 and S100 positive staining at $20 x$ magnification.

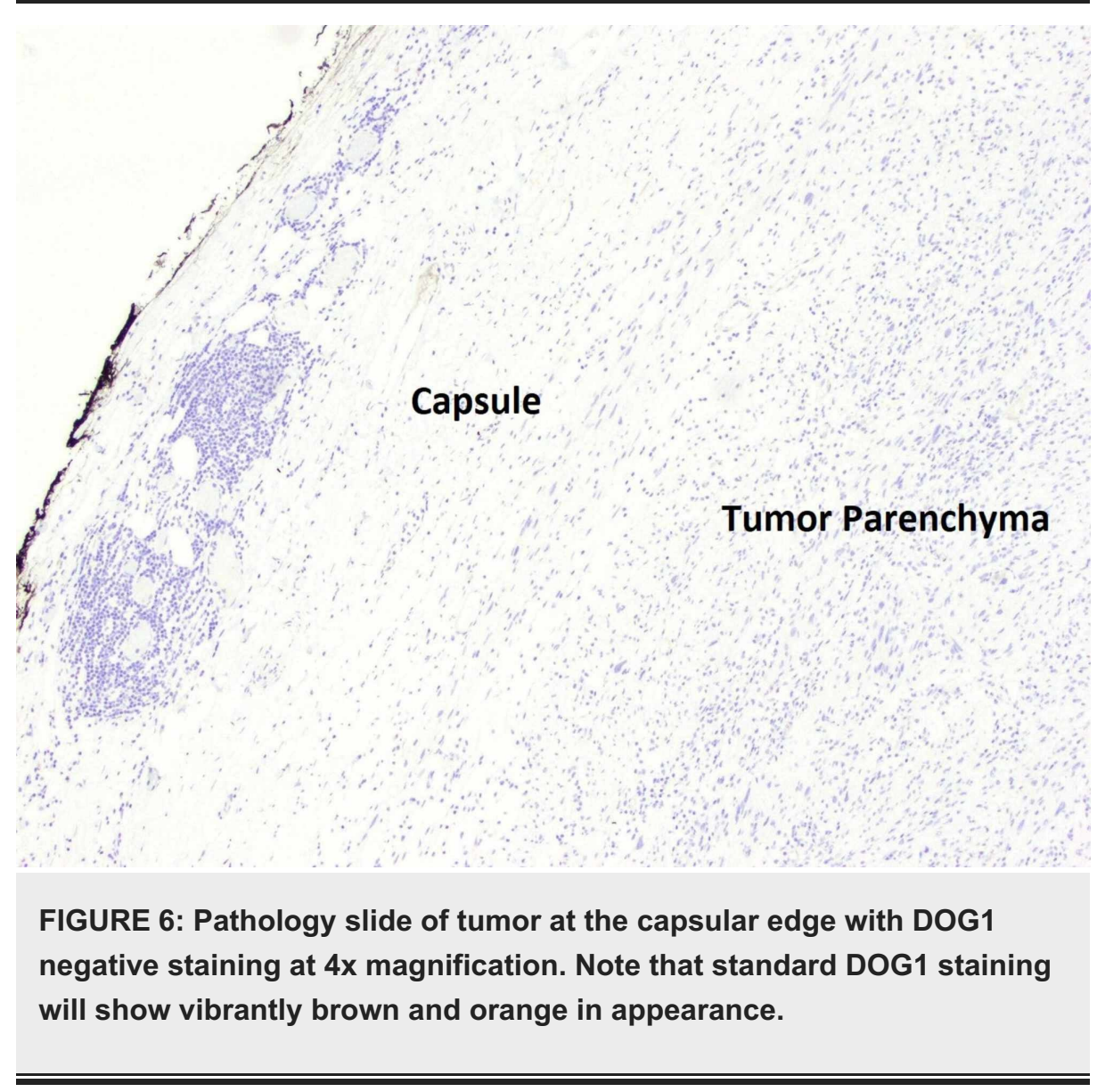




\section{Cureus}

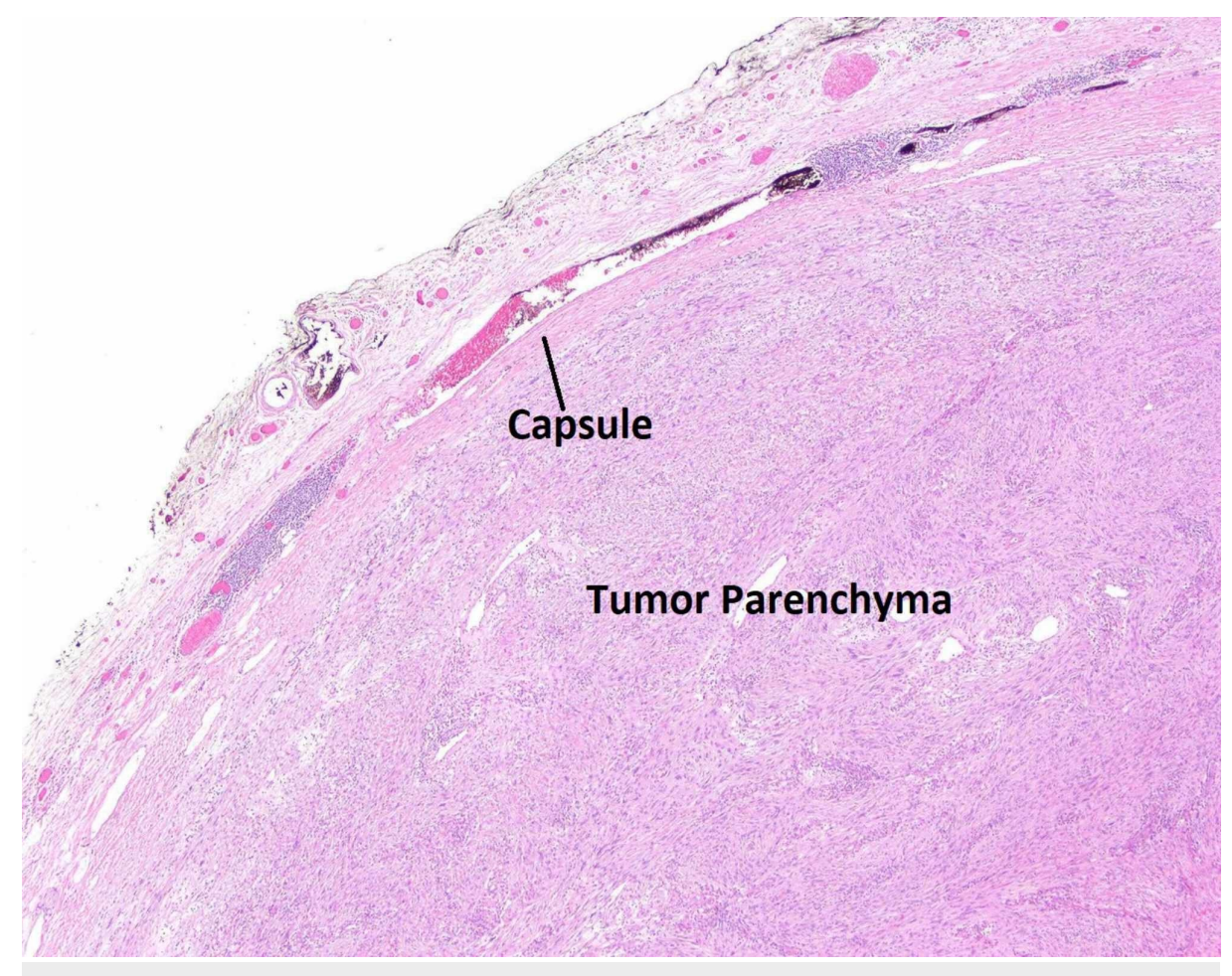

FIGURE 7: Pathology slide of tumor with haematoxylin and eosin staining at $4 x$ magnification. Note that the tumor does not invade into the capsule, which was consistently noted in all other slides examined.

\section{Discussion}

Schwannomas are tumors derived from schwann cells. GI schwannomas are very rare and account for approximately $5 \%$ of all mesenchymal tumors. GI schwannomas are more often found within the Auerbach's myenteric plexus and less likely to be found in the Meissner's submucosal plexus [1]. Mostly commonly found in the stomach, GI schwannomas are less frequently found in the rectum, colon, small intestine, or esophagus $[4,5]$. Within the colorectal tract, schwannomas typically present in the cecum, right colon, and sigmoid with relatively equally found frequency. Transverse colon schwannomas were found in only $5 \%$ of literature cases of all colorectal schwannomas [5].

In a systematic literature review of schwannomas of the colon and rectum published by Bohlok et al., colorectal schwannomas occurred slightly more often in female patients (58\%) and had a mean age of onset over the age of 60 years. In their 95-patient study examining colonic or rectal schwannoma, a large portion of patients were asymptomatic on diagnosis (36\%), but others presented with rectorrhagia, abdominal pain, or constipation [5]. Other studies noted that GI schwannomas can manifest as colonic obstructions or as intussusception [6-8].

Most GI schwannomas are incidental findings during screening colonoscopy or imaging. During colonoscopy, an incidentally found GI schwannoma appears as a submucosal mass whose overlying mucosa is either smooth in appearance or has an ulceration over the mass [3-5]. When found with CT imaging, it appears as an exophytic mass with homogenous enhancement. Cystic changes, necrosis, or calcifications within tumors are not commonly found [4]. A preoperative diagnosis is challenging, as endoscopic mucosal biopsy of these lesion provides limited incite that could help differentiate these masses from other mesenchymal tumors of the GI tract such as gastrointestinal stromal tumors (GIST), neuroendocrine tumors, leiomyomas, or leiomyosarcomas [5,9]. GISTs are the most common differential diagnosis [10].

A definitive diagnosis is made on immunohistopathological examination of the resected specimen. Macroscopic examination of these tumors will show a well-defined tumor with many lobule appearing areas (Figure 7) that may have an ulceration in their mucosa [11]. Schwannomas stain positive for S100 (Figures 4,5) and infrequently for vimentin. They stain negative for several markers that help differentiate these tumors from other mesenchymal cells such as DOG1 (Figure 6), SMA, desmin, CD 117, p53, CD-34 and c-kit [10, 12]. Most GISTs are S-100 negative, but CD-34 and c-kit positive [10, 13-16].

Microscopically, schwannomas consist of sheets of fusiform cells with little nuclear pigmentation and a low rate of mitosis (Figure 3). Besides simple microscopic examination and protein staining, pathologists rely on 
Antoni A and Antoni B classifications, two histological growth patterns that schwannomas demonstrate [2]. Named after the Swedish neuropathologist, Nils Ragnar Eugene Antoni, this system characterizes the two most common forms of growth that schwannomas display. Antoni A histology notes Verocay bodies, fusiform cells densely packed in a palisade formation. Antoni B histology shows a looser group of fusiform cells with oblong or round nuclei seated in xanthomatous histiocytes and myoid stroma [2]. The tumor in this case study was likely Antoni A due to its dense formation of fusiform cells.

Ninety-eight percent of colorectal schwannomas are benign [5]. Two histopathological markers of importance are the Ki-67 index and the mitotic index, both of which are strong predictors for malignancy. A Ki-67 index greater than 5\% can correlate to malignancy and any tumor with greater than $10 \%$ is considered malignant. A mitotic index of greater than 5 mitotic figures per high power fields is also indicative of a risk for malignancy [9-10]. In Bohlok et al.'s systematic review, three out of 93 cases of colorectal schwannomas were malignant [5]. Their study, however, lacked data on mitotic activity, as well as marker studies for Ki67. In other case reports describing malignant colorectal schwannomas, malignancy was dependent on the size of the tumor and the number of mitoses present in their specimen, in addition to long-term local recurrence and liver metastasis [17-20].

Complete surgical resection with tumor-free margins is believed to be the best therapeutic option [6]. Tumor recurrence is generally owed to incomplete surgical resection and/or inadequate margins. Adjuvant therapies are not commonly recommended, if surgical resection is able to achieve negative margins [9]. Because of its rarity and the challenge of producing a preoperative diagnosis, review of the clinical features of the disease would be of benefit for surgeons and clinicians who encounter colorectal schwannomas.

\section{Conclusions}

Colorectal schwannomas are a very rare form of benign GI schwannomas that affect mostly women and those over the age of 60 . Most cases of these schwannomas are incidentally found during screening colonoscopy and present as submucosal masses. Biopsies do not provide an appropriate diagnosis and surgical resection with tumor negative margins is typically needed for diagnosis and treatment. Immunohistochemistry staining, specifically S-100 tumor marker, will best elucidate the appropriate diagnosis of a schwannoma.

\section{Additional Information \\ Disclosures}

Human subjects: Consent was obtained by all participants in this study. Conflicts of interest: In compliance with the ICMJE uniform disclosure form, all authors declare the following: Payment/services info: All authors have declared that no financial support was received from any organization for the submitted work. Financial relationships: All authors have declared that they have no financial relationships at present or within the previous three years with any organizations that might have an interest in the submitted work. Other relationships: All authors have declared that there are no other relationships or activities that could appear to have influenced the submitted work.

\section{References}

1. Tsunoda C, Kato H, Sakamoto T, et al.: A case of benign schwannoma of the transverse colon with granulation tissue. Case Rep Gastroenterol. 2009, 3:116-120. 10.1159/000214837

2. Nonose R, Lahan AY, Santos Valenciano J, Martinez CA: Schwannoma of the colon. Case Rep Gastroenterol. 2009, 3:293-299. 10.1159/000237736

3. Baig MMAS, Patel R, Kazem MA, Khan A: Schwannoma in the ascending colon, a rare finding on surveillance colonoscopy. J Surg Case Rep. 2019, 2019:46. 10.1093/jscr/rjz046

4. Mekras A, Krenn V, Perrakis A, et al.: Gastrointestinal schwannomas: a rare but important differential diagnosis of mesenchymal tumors of gastrointestinal tract. BMC Surg. 2018, 18:47. 10.1186/s12893-0180379-2

5. Bohlok A, El Khoury M, Bormans A, et al.: Schwannoma of the colon and rectum: a systematic literature review. World J Surg Oncol. 2018, 16:125. 10.1186/s12957-018-1427-1

6. González Ruiz Y, Reyes Delgado A, Guiterrez Alonso C, Franco Rubio JI, González Herrero M: Sigmoid intussusception as a clinical presentation of colonic schwannoma: pediatric case (Article in Spanish). Arch Argent Pediatr. 2019, 117:68-71. 10.5546/aap.2019.e68

7. Wilde BK, Senger JL, Kanthan R: Gastrointestinal schwannoma: an unusual colonic lesion mimicking adenocarcinoma. Canadian J Gastroenterol Hepatol. 2010, 24:233-236. 10.1155/2010/943270

8. Wang WB, Chen WB, Lin JJ, Xu JH, Wang JH, Sheng QS: Schwannoma of the colon: a case report and review of the literature. Oncol Lett. 2016, 11:2580-2582. 10.3892/ol.2016.4271

9. Uhr A, Singh AP, Munoz J, et al.: Colonic schwannoma: a case study and literature review of a rare entity and diagnostic dilemma. Am J Surg. 2016, 82:1183-1186.

10. Çakır T, Aslaner A, Yaz M, Gündüz UR: Schwannoma of the sigmoid colon. BMJ Case Rep. 2015, 2015:2014208934. 10.1136/bcr-2014-208934

11. Das Gupta TK, Brasfield RD: Tumors of peripheral nerve origin: benign and malignant solitary schwannomas. CA Cancer J Clin. 1970, 20:228-233. 10.3322/canjclin.20.4.228 


\section{Cureus}

12. Fletcher CD, Berman JJ, Corless C, et al.: Diagnosis of gastrointestinal stromal tumors: a consensus approach. Hum Pathol. 2002, 33:459-465. 10.1053/hupa.2002.123545

13. Inagawa S, Hori M, Shimazaki J, et al.: Solitary schwannoma of the colon: report of two cases . Surg Today. 2001, 31:833-838. 10.1007/s005950170060

14. Miettinen M, Virolainen M, Maarit-Sarlomo-Rikala: Gastrointestinal stromal tumors--value of CD34 antigen in their identification and separation from true leiomyomas and schwannomas. Am J Surg Pathol. 1995, 19:207-216. 10.1097/00000478-199502000-00009

15. Ramai D, Lai J, Changela K, Reddy M, Shahzad G: Transverse colon schwannoma treated by endoscopic mucosal resection: a case report. Mol Clin Oncol. 2017, 7:830-832. 10.3892/mco.2017.1418

16. Hornick JL, Bundock EA, Fletcher CDM: Hybrid schwannoma/perineurioma: clinicopathologic analysis of 42 distinctive benign nerve sheath tumors. Am J Surg Pathol. 2009, 33:1554-1561. 10.1097/PAS.0b013e3181accc6c

17. Schwartz DA: Malignant schwannoma occurring with Schistosoma japonicum: a case report . Southeast Asian J Trop Med Public Health. 1982, 13:601-605.

18. Catania G, Puleo C, Cardì F, Catalano F, Iuppa A, Buffone A: Malignant schwannoma of the rectum: a clinical and pathological contribution. Chir Ital. 2011, 53:873-877.

19. Bodner E, De los Santos EV: The malignant degeneration of a rectal neurinoma; "views on the tendency of the tumors of the nerve sheaths towards malignant degeneration". Philipp J Surg Spec. 1965, 20:125-141.

20. Wang CL, Neville AM, Wong TZ, Hall AH, Paulson EK, Bentley RC: Colonic schwannoma visualized on FDG PET/CT. Clin Nucl Med. 2010, 35:181-183. 10.1097/RLU.0b013e3181cc632a 